\title{
Envejecimiento de la piel: influencia del tabaco en mujeres privadas de libertad en centro penitenciario en Portoviejo, Ecuador
}

\section{Efectos del tabaquismo en el envejecimiento de la piel}

QF, Mirella Adum Lípari .Mg. $\left({ }^{1}\right)$

Tec. Helen Jhosoá Agrazal Vásquez $\left(^{2}\right)$

$\left({ }^{1}\right)$ Universidad San Gregorio, Portoviejo Ecuador

$\left({ }^{2}\right)$ Universidad San Gregorio, Portoviejo Ecuador

Contacto: mnadum@hotmail.com

Receptado: 08/10/2015 Aceptado: 14/12/2015

\section{Resumen}

La salud de la piel es importante no sólo por su aspecto, si no por el papel que tiene en la realización de muchas tareas corporales esenciales. Esta juega un rol vital como primera línea de defensa del cuerpo contra la infección y otros elementos duros. Dentro de la afecciones de la piel es de valiosa importancia la atención al envejecimiento prematuro de la misma. La investigación tuvo como objetivo general determinar la influencia del tabaco en mujeres privadas de libertad. Para este, se fijó como objeto de estudio la salud de la piel y se tomó como campo de observación el Centro de Rehabilitación Femenino y Centro de Detención Provisional Tomás Larrea en la Ciudad de Portoviejo, Ecuador. Para el desarrollo del trabajo, se utilizaron varios soportes metodológicos como la empleo de tres métodos fundamentales: el inductivodeductivo el científico y el analítico- sintético, los cuales fueron desarrollados a través de diferentes técnicas que permitieron dilucidar varias interrogantes sobre la temática. Se concluyó sobre la nocividad de la adicción al tabaquismo, la cual es la causante de muchas enfermedades, donde se incluye el envejecimiento prematuro de la piel y los efectos perjudiciales tanto estéticos como para la salud de las mujeres: Se pudo concluir, además, sobre la existencia de situaciones socioeconómicas que demuestran las realidades con las cuales conviven las personas privadas de libertad en ese centro. 
Rev. SINAPSIS, Vol. 7, № 2, diciembre 2015

Palabras clave: Envejecimiento de la piel, tabaquismo, consumo del tabaco, mujeres privadas de libertad

The skin aging: snuff influence on female inmates in prison in Portoviejo, Ecuador

\begin{abstract}
The health of the skin is important not only for its appearance but by the role it plays in the realization of many essential bodily tasks. This plays a vital role as the first line of defense of the body against infection and other harsh elements. Among the skin conditions great attention is paid to importance of premature aging thereof. The overall objective of research was to determine the influence of snuff in women prisoners. For this, it was set as an object of study the health of the skin and it was taken as the field observation "el Centro de Rehabilitación Femenino y Centro de Detención Provisional Tomás Larrea en la Ciudad de Portoviejo, Ecuador". There were used the scientific analytic and synthetic methods, which were developed through different techniques that allowed to elucidate some questions on the theme. It was concluded on the harmfulness of smoking addiction, which the cause of many diseases, including the skin aging is affecting not only aesthetically but also the health of women including harmful effects.
\end{abstract} Keywords: Skin aging, smoking, consumption of snuff, prisoners

\title{
Introducción
}

El tabaquismo es un problema que se presenta en todo el mundo, "hasta ahora el uso del tabaco se ha asociado a más de veinticinco enfermedades" (Álvarez, 2013. p. 32).Cabe recalcar que no existe un número exacto de consumidores tanto en países desarrollados como en los subdesarrollados. A pesar de que el consumo de tabaco es perjudicial; no se ha logrado desarrollar un plan para erradicarlo completamente, por lo tanto es la adicción que una persona presenta hacia el tabaco provocado por uno de sus componentes activos principalmente la nicotina, ya que actúa como antidepresivo y alivio sintomático de la ansiedad; es así que la acción de dicha sustancia termina condicionando el abuso de consumo, esta adicción causa enfermedades nocivas para la salud del consumidor. 
Se ha comprobado que el tabaco es perjudicial en todos los países del mundo, se ha creado campañas para su erradicación, pero desgraciadamente no ha tenido buenos resultados. El tabaquismo en Ecuador en los últimos tiempos ha sido el causante de muertes y más aún de provocar envejecimiento prematuro en personas a edades tempranas, lo que ocasiona gran incidencia en problemas cutáneos de la población en general.

Se ha identificado el tabaquismo como causa de enfermedades del corazón, cáncer y enfermedades respiratorias. La piel no está excepta de los efectos nocivos producidos por el tabaquismo, es así que la piel de la persona que fuma y aquellos que indirecta y pasivamente aspiran las sustancias tóxicas proveniente del tabaco, se observa envejecida y con mayor cantidad y profundidad de arrugas, especialmente alrededor de los labios y ojos, también se ha observado la presencia de acné no inflamatorio, dolencia por presencia de poros cerrados, quistes y comedones abiertos y cerrados en fumadores. Las personas privadas de la libertad están expuestas a esta problemática, y es precisamente en el Centro de Rehabilitación Social Femenino y Centro de Detención Provisional Tomás Larrea donde se enfoca la investigación realizada.

La investigación se enfocó sobre la problemática de cómo influye consumo de tabaco en el envejecimiento de la piel de las mujeres privadas de la libertad del Centro de Rehabilitación Social Femenino y Centro de Detención Provisional Tomás Larrea de Portoviejo.

Éste estudio tuvo entre sus principales líneas concienciar a la comunidad penitenciaria femenina de lo perjudicial que resulta el consumo del tabaco en el envejecimiento prematuro de la piel y la presencia del denominado rostro del fumador, el cual tiene una característica marcada de piel rugosa, grisácea, relieves óseos en los pómulos, los labios y los ojos se encuentran rodeados de arrugas finas, líneas profundas y superficiales en las mejillas y las mandíbulas, dientes amarillos y dedos amarillos. López Barrí en su artículo "El tabaquismo: consecuencias en dermatología y estética" aborda los efectos del tabaco en la disminución en los niveles de vitamina A, produciendo cambios en la calidad y cantidad del colágeno, elastina, la aparición de la atrofia dérmica y la disminución de fibroblastos, así como el acortamiento de los capilares (López, 2014). 
Rev. SINAPSIS, Vol. 7, No 2, diciembre 2015

En ocasiones se muestra carencia de vitamina A en personas fumadoras y con una alimentación inadecuada debido al bajo consumo de alimentos de origen animal, como la leche, la carne, el huevo y otros. Esta deficiencia vitamínica puede ocasionar hiperqueratosis o piel seca o escamosa.

En el trabajo se aborda el estudio realizado en un centro penitenciario de mujeres donde se observó la muestra de un grupo de fumadoras, donde se pudo visualizar que la adicción al tabaco es bastante propagado en la población general, pero que este aumenta marcadamente en situaciones de privaciones asociadas fundamentalmente las consecuencias negativas de la pérdida de libertad

\section{Materiales y métodos}

La influencia del consumo del tabaco en el envejecimiento de la piel pudo corroborarse en la investigación mediante el uso de tres métodos fundamentales:

El método Inductivo - Deductivo, el cual permitió en una primera etapa investigativa la inducción de los principios explicativos a partir de los fenómenos observados que sirvieron para llegar a una segunda fase en la indagación. Esta metodología se centró primeramente en un primer momento que consistió en la creación de un cuerpo teórico donde se tomaron los principios elementales y los fenómenos observables sobre la influencia del tabaco en el envejecimiento de la piel en las reclusas. La segunda etapa consistió la deducción las leyes generales que conducen al fenómeno planteadas en el cuerpo teórico, válidas para explicar dicho fenómeno.

Para la puesta en marcha de este trabajo fue de gran utilidad el método Científico, el cual permitió la producción de conocimientos en la ciencias, se basó en lo empírico y medición, sujeto a principios específicos de las pruebas de razonamiento.

Fue de gran importancia el método Analítico - Sintético mediante este se podo concluir sobre la realidad del fenómeno observado. Este fue válido para el análisis por separado de los elementos y las causas que intervienen el en la realización del fenómeno del tabaquismo en la mujeres en dicho centro penitenciario. 
La efectividad de estos tres métodos fue posible por la materialización de diferentes técnicas de investigación: la observación directa, que se hizo de forma sistemática, consciente, planificada y objetiva. Esta se utilizó para obtener la información primaria acerca de la cárcel que sirvió como campo estudio. Esta técnica permitió conocer el proceder y la conducta de las mujeres incluidas en el objeto de estudio durante un tiempo previo a la obtención de resultados. Después se realizaron entrevistas de forma cualitativa y estructura, las cuales posibilitaron la recopilación de información detallada sobre el tema del tabaquismo a las mujeres en dicho recinto penitenciario. Finalmente fueron de gran utilidad en la corroboración de los resultados, las encuestas que sentaron bases sólidas para obtener una aproximación a la realidad vivencial del proyecto.

\section{Resultados}

Se tomó como problema investigativo, la magnitud en que influye el uso excesivo del tabaco en las personas privadas de la libertad en el Centro de Rehabilitación Social Femenino y Centro de Detención Provisional Tomás Larrea de Portoviejo. Esta problemática fue investigada en el tema sobre influencia del tabaco en el envejecimiento de la piel de las personas privadas de la libertad en dicha institución.

El trabajo se centró en la introducción de resultados reales a través de la solución a varias interrogantes que permitieron conocer cuáles son los efectos del consumo de tabaco sobre la piel de las mujeres privadas de la libertad del Centro de Rehabilitación Social Femenino y Centro de Detención Provisional Tomás Larrea de Portoviejo.

A través de un proceso primeramente documental, que permitió llegar a una segunda fase observacional, se pudo dilucidar la solución a diferentes interrogantes sobre un problema dado en un tema de relevante importancia social. Las preguntas investigativas fueron de vital importancia en el proceso de aplicación del método científico. Estas se formularon de forma lógica a una población de 70 mujeres, donde se prestó mayor atención a 36 de ellas, las cuales eran consumidoras activas de tabaco.

Se formularon 8 preguntas de investigación descriptivas, que sirvieron para la representación de las variables fundamentales estudiadas, la edad y nivel de consumo de tabaco. 
Para la obtención de resultados reales, se hizo inicialmente un estudio referencial sobre los componentes del tabaco. Dichos resultados bibliográficos obtenidos partieron del estudio de algunas de las sustancias químicas asociadas con el humo del tabaco. Compuestos, tales como el amoniaco, el arsénico, el butano, el cianuro, el formaldehído, el metano, monóxido de carbono. La mayor parte de las sustancias en el humo del tabaco son causantes de efectos nocivos dentro de los cuales se encuentra el envejecimiento de la piel.

El estudio corroboró que el consumo del tabaco por parte de las mujeres del centro penitenciario son susceptibles a efectos negativos corporales como: el envejecimiento precoz, la adicción, y la aparición de acné. Además, la experiencia permitió concienciar a la comunidad de lo perjudicial que resulta el consumo del tabaco en el envejecimiento prematuro de la piel y la presencia del denominado rostro del fumador, el cual tiene una característica marcada de piel rugosa, grisácea, y relieves óseos en los pómulos.

Este estudio observacional permitió asentir que las personas consumidoras de tabaco presentan arrugas causadas por dos razones. La primera por qué los cigarrillos contienen sustancias nocivas para la piel y segundo solo por el simple hecho de fumar se provoca un aumento de contracciones y gestos alrededor de la boca que se convierten en arrugas, que en palabras técnicas se denominan arrugas periorales y popularmente código de barras, sin dejar de mencionar la aparición temprana de las patas de gallo o arrugas orbiculares; por lo tanto, las personas privadas de la libertad fumadores y quienes están expuestos al humo, tienden a cerrar los ojos involuntariamente para evitarlo, lo que genera una mayor aparición de pliegues en el rostro.

Se trabajó con un cuestionario de 8 preguntas prefijadas con el objetivo cerrado y único en forma de encuesta, que consistía en establecer una evaluación práctica del efecto del consumo del tabaco en la piel de las mujeres de dicho centro penitenciario.

La encuesta se estructuró tomando como punto inicial las características físicas que presentan las internas que consumen tabaco. Se tomó como fuente de referencia en todos los casos las personas privadas de la libertad del Centro de Detención Provisional de Portoviejo 
Rev. SINAPSIS, Vol. 7, № 2, diciembre 2015

En este apartado se comenzó con la indagación de la variable continua de la edad, como una de las variables más relevantes en el estudio de una población determinada. Esta interrogante pretendió un acercamiento a los tres grandes grupos de edades planteados por Sauvy quien propuso que para un análisis social se deben considerar tres grandes grupos de edades que agrupan a jóvenes, adultos y viejos.

\begin{tabular}{|c|c|c|}
\hline Alternativas & Frecuencia & Porcentaje \\
\hline $18-30$ años & 34 & 48 \\
\hline $31-50$ años & 27 & 39 \\
\hline $51-65$ años & 9 & 13 \\
\hline Total & 70 & 100 \\
\hline
\end{tabular}

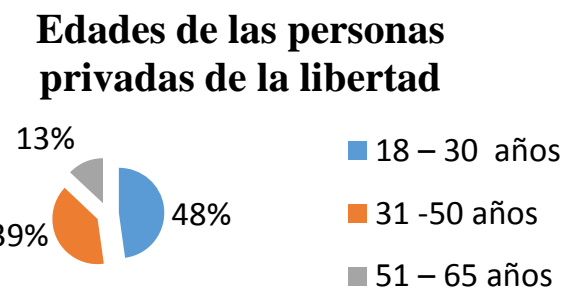

Las mujeres encuestadas debían consignar en cuál de los tres grupos se encontraba. Los resultados se enunciaron de la siguiente manera:Cuadro 1 Gráfico 1

En el cuadro y gráfico No1, se evidencia que las personas privadas de la libertad en el momento en que se realizaron las respectivas encuestas, en su mayoría varían en edades comprendidas entre los 18-30 años con un 48\%; entre los 31-50 años el 39\%; y entre los 51-65 años únicamente el 9\%.

Como se evidencia, las edades más frecuentes de las mujeres privadas de la libertad que se encuentran en el Centro de Detención Provisional, varía entre los 18 y 30 años, debido a que hasta este lugar solo llegan aquellas mujeres mayores de edad, puesto que los menores de edad son derivadas directamente al correccional de menores y las adultas mayores reciben arresto domiciliario.

La segunda interrogante versó sobre el aspecto concerniente al consumo del tabaco. 


\section{Cuadro 2}

\begin{tabular}{|l|l|l|}
\hline Alternativas & Frecuencia & Porcentaje \\
\hline Sí & 36 & 51 \\
\hline No & 34 & 49 \\
\hline Total & 70 & 100 \\
\hline
\end{tabular}

\section{Gráfico 2}

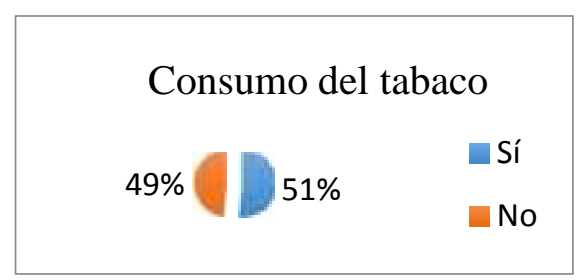

En las ilustraciones se indica que las mujeres privadas de la libertad en el Centro de Detención Provisional de Portoviejo en un 51\% consumen tabaco; mientras tanto el $49 \%$ no consume.

Al momento de realizar la encuesta en las mujeres privadas de la libertad, se notó que no eran lo suficientemente sinceras, puesto que solo un poco más de la mitad aseguraron consumir tabaco, no obstante quienes negaron esta adicción presentaban rasgos evidentes del consumo de tabaco y otras sustancias. Esto sucede por la prohibición de las autoridades del Centro en no permitir fumar, lo que realizan a escondidas las internas.

En la tercera pregunta encuestada se estableció una relación con la interrogante previamente formulada. Toda vez que las encuestadas se hubiesen consignado como fumadoras debían establecer sus edades en que comenzaron la práctica del consumo del tabaco.

\section{Cuadro 3}

\begin{tabular}{|c|c|c|}
\hline Alternativas & Frecuencia & Porcentaje \\
\hline $10-20$ años & 22 & 61 \\
\hline $21-30$ años & 8 & 22 \\
\hline $31-40$ años & 4 & 11 \\
\hline $41-45$ años & 2 & 6 \\
\hline Total & 36 & 100 \\
\hline
\end{tabular}

\section{Gráfico 3}



En el cuadro y gráfico No3, se manifiesta las edades de inicio del consumo de tabaco en mujeres privadas de la libertad que se encuentran retenidas en el Centro de Detención Provisional de Portoviejo; estas edades varían de 10-20 años el 61\%; de 21-30 años el 22\%; de 31-40 años el 11\% y de 41-45 años el 6\%.

En su mayoría las mujeres privadas de la libertad aseguraron que empezaron a consumir tabaco desde los 10 años de edad. Esto indicaría que el problema empieza en el hogar 
Rev. SINAPSIS, Vol. 7, № 2, diciembre 2015

debido a la situación socio económico en donde ellas crecieron, en una familia disfuncional, con problemas económicos y sociales muy arraigados.

La pregunta cuatro discurrió sobre la frecuencia del consumo del tabaco por parte de las mujeres fumadoras en esta institución carcelera.

\section{Cuadro 4}

\begin{tabular}{|l|c|c|}
\hline \multicolumn{1}{|c|}{ Alternativas } & Frecuencia & Porcentaje \\
\hline A diario & 24 & 67 \\
\hline $\begin{array}{l}\text { Dos o tres veces } \\
\text { por semana }\end{array}$ & 7 & 19 \\
\hline Ocasionalmente & 5 & 14 \\
\hline Total & 36 & 100 \\
\hline
\end{tabular}

\section{Gráfico 4}

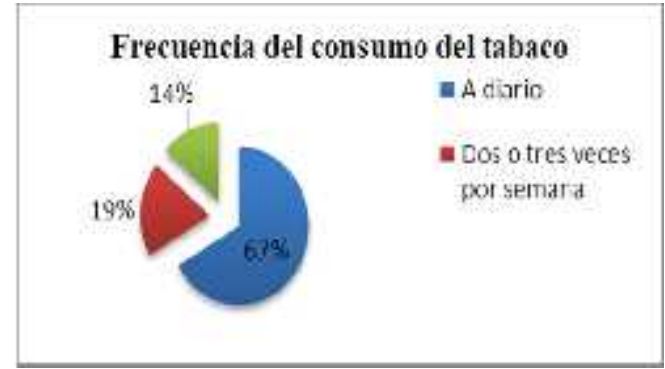

En el cuadro y gráfico No4, Se muestra que el 67\% fuma a diario; $19 \%$ fuma dos o tres veces por semana y el $14 \%$ fuma ocasionalmente; con los porcentajes expuestos se puede evidenciar que en su mayoría las personas privadas de la libertad consumen tabaco.

Las personas que consumen tabaco lo realizan a diario, siendo así una adicción que no pueden controlar, debido al estrés que ocasiona el encierro en dicho lugar.

La investigación consideró necesario en la observación establecer una relación entre el consumo de cigarrillos y el grado de aparición de posibles afecciones. Para ello se decidió plantearse una quinta pregunta, cuyo objetivo fue determinar la cantidad del consumo diario de cigarrillos por día en dichas mujeres.

\section{Cuadro 5}

\begin{tabular}{|l|c|c|}
\hline \multicolumn{1}{|c|}{ Alternativas } & Frecuencia & Porcentaje \\
\hline 1 - 5 por día & 22 & 61 \\
\hline 6 - 10 por día & 9 & 25 \\
\hline $11-15$ por día & 3 & 8 \\
\hline $16-20$ por día & 1 & 3 \\
\hline $21-30$ por día & 1 & 3 \\
\hline
\end{tabular}

\section{Gráfico 5}

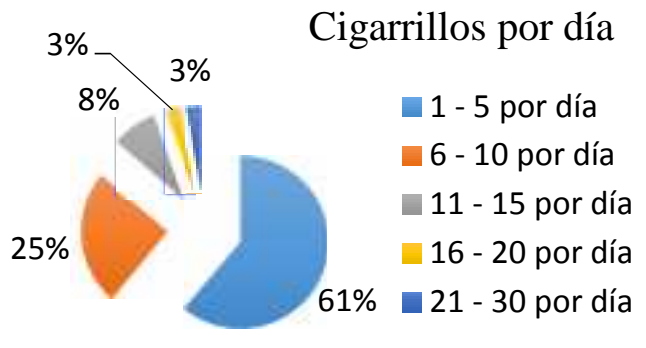


Rev. SINAPSIS, Vol. 7, № 2, diciembre 2015

ISSN 1390 - 9770

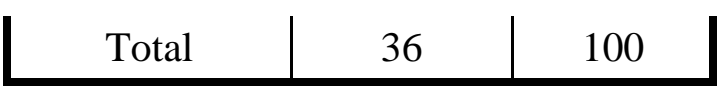

En el cuadro y gráfico No5, se indica que el $61 \%$ consume de 1-5 cigarrillos por día; el 25\% de 6-10 cigarrillos por día; el 8\% de 11-15 cigarrillos por día; el 3\% de 16-20 cigarrillos por día y el 3\% de 21-30 cigarrillos por día.

Es frecuente que las mujeres privadas de la libertad fumen de 1 a 5 cigarrillos diarios, esto respalda los resultados del cuadro anterior.

La sexta cuestión indagada en la encuesta buscaba una panorámica sobre las mujeres que en algún momento habían dejado de formar, las cifras se comportaron de la siguiente manera:

\section{Cuadro 6}

\begin{tabular}{|c|c|c|}
\hline Alternativas & Frecuencia & Porcentaje \\
\hline Sí & 27 & 75 \\
\hline No & 9 & 25 \\
\hline Total & 36 & 100 \\
\hline
\end{tabular}

\section{Gráfico 6}

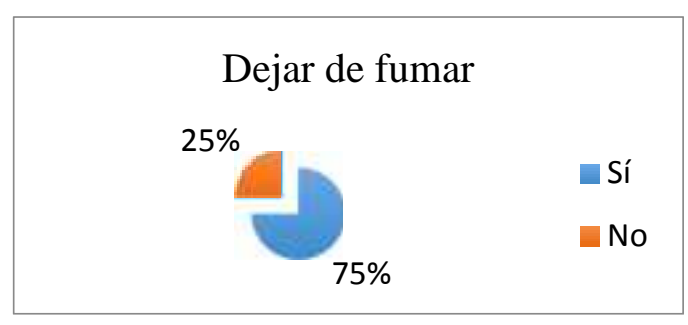

Como se observa en el cuadro y gráfico 6 , se exterioriza que el $75 \%$ de las mujeres privadas de la libertad que se encuentran en el Centro de Detención Provisional de Portoviejo sí ha dejado alguna vez el tabaco; el 25\% nunca ha dejado de consumir el tabaco.

En su mayoría mencionaron que alguna vez en su vida se propusieron dejar el tabaco, lo consiguieron por un período corto, pero por razones de ansiedad que viven en el Centro nuevamente retomaron el hábito de fumar.

La séptima pregunta en cuestión examinaba sobre deseos y pretensiones de dejar de fumar, los resultados se arrojan en el siguiente cuadro y gráfico. 
Rev. SINAPSIS, Vol. 7, № 2, diciembre 2015

Cuadro 7

\begin{tabular}{|c|c|c|}
\hline Alternativas & Frecuencia & Porcentaje \\
\hline Sí & 30 & 83 \\
\hline No & 6 & 17 \\
\hline Total & 36 & 100 \\
\hline
\end{tabular}

\section{Gráfico 7}

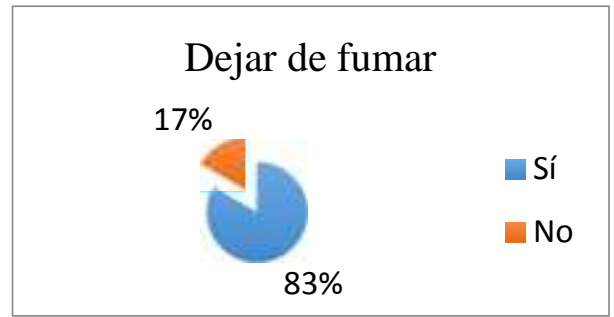

En el cuadro y gráfico No 7, Se revela que el 83\% sí desea dejar de fumar; y el 17\% asegura que no les gustaría dejar de fumar.

En su gran mayoría las mujeres privadas de la libertad aseguran que si quieren dejar de fumar por los daños que en el futuro ocasiona este hábito, pero dicen no encontrar la ayuda psicológica y social necesaria para dejar de consumir tabaco.

Pregunta No8. ¿Ha notado cambios en su piel, que usted le atribuya al consumo del cigarrillo.

Se formuló en dicha encuesta esta pregunta conclusiva que pretendía obtener en estado de opinión en relación a los efectos del consumo de tabaco en la piel de la mujeres 36 mujeres evaluadas como fumadoras en el campo investigativo.

\section{Cuadro 8}

\section{Gráfico 8}

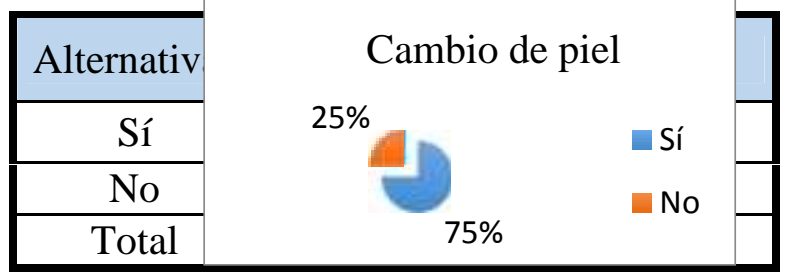

En estas últimas ilustraciones se hace constar que dentro del $57 \%$ sí ha notado

cambios en la piel atribuido al consumo del tabaco y el $43 \%$ no manifiesta cambios en su piel.

Esta situación es imperceptible para las internas, ellas no asocian los cambios en su piel con el tabaco, el envejecimiento prematuro lo asumen como normal. Hay que destacar que una pequeña mayoría de internas contestaron que si se dan cuenta, a pesar que los cambios en la piel son evidentes.

\section{Discusión.}


Rev. SINAPSIS, Vol. 7, № 2, diciembre 2015

Branco (2010) concluyó sobre cómo el tabaco se considera un factor ambiental que contribuye de forma importante al envejecimiento prematuro de la piel. Este planteó acerca del incremento de las arrugas faciales y color ceniza grisáceo en esta.

La investigación corrobora algunas aserciones hechas por estudiosos del tema. En diversos trabajos se ha comprobado que con administración sistémica de nicotina, estos plantean que la cicatrización de las heridas empeoraba en los fumadores, lo que demuestra una relación entre el número de paquetes fumados por días y la aparición de necrosis postoperatorias. La explicación de estos hechos se atribuye al efecto vasoconstrictor de la nicotina.

De esta manera Cornejo (2014) aseveró que fumar un cigarrillo produce vasoconstricción durante 90 minutos y, por fumar un cigarrillo, el flujo de sangre digital llega a disminuir un $42 \%$. En conclusión, este señaló que antes de un acto quirúrgico y después del mismo no se debe fumar.

Si se hace un análisis comparativo sobre uno de los trabajos Becoña (2010):

El tabaco altera el equilibrio entre la elastasa y su mayor regulador, el inhibidor de la alfa 1-proteinasa, lo que favorece la ruptura de las fibras elásticas que soportan la piel y le dan resistencia...la acumulación de desechos de elastina en la dermis va seguida de una degeneración del colágeno que la rodea, distorsionando la piel y facilitando la formación de arrugas.

La investigación permitió concluir que el contacto que el cutis mantiene con el humo del cigarrillo provoca que la piel sufra asfixia celular y por ende se sufra de cutis grisáceo; siendo así que el depósito de humo en los poros provoca dilatación de los mismos y aparición de puntos negros.

En dicho campo investigativo se evaluó que las mujeres que fuman poseen tres veces más probabilidades de presentar arrugas en su piel a diferencia de aquellas que no fuman, a esto se le denomina arrugas prematuras debido a la disminución de la hidratación del estrato córneo de la cara que provoca el humo del tabaco. 
En una de las cuestiones encuestadas se demostró que un \% de mujeres afirman de la manera en que el consumo del tabaco afecta de alguna forma la piel. Es decir el tabaco altera el equilibrio entre la elastasa y su mayor regulador, el inhibidor de la alfa 1proteinasa, con un incremento de la liberación de la elastasa por los neutrófilos y los macrófagos y una inactivación por oxidación de su inhibidor. Todo ello ocasiona un incremento de la actividad de la metaloproteinasas en la piel, rompiendo las fibras elásticas que soportan la piel y le confieren su resistencia. La acumulación de desechos de elastina en la dermis va seguida de una degeneración del colágeno que la rodea.

Se asume recomendar sobre la necesidad que las internas del Centro de detención provisional accedan a material bibliográfico para enriquecer más sus conocimientos acerca de los aspectos negativos que se relacionan con el consumo del tabaco y que conozcan que es una de las causas principales que provoca la muerte.

Se debe programar además, realizar periódicamente actividades que permitan conocer el porcentaje de internas fumadoras para verificar que esta cantidad es disminuida o aumentada. Es necesario que las autoridades realicen un chequeo médico continuamente para verificar cuales son los efectos causados en la piel de las internas a causa del consumo.

Se debe difundir el material educativo elaborado a raíz de la investigación, el cual contiene información relevante acerca de los efectos negativos del consumo del tabaco y lo perjudicial que resulta el uso excesivo, entre las internas, familiares y personal que labora en el lugar de estudio.

\section{Conclusiones}

Se concluyó que el consumo del tabaco puede causar enfermedades del corazón, cáncer, enfermedades respiratorias, envejecimiento prematuro de la piel evidenciándose en sus consumidores una piel grisácea con mayor cantidad de arrugas superficiales y profundas, especialmente alrededor de labios y ojos y quienes lo consumen son personas que se dejan llevar por el estrés, afectando no solo a quienes han adquirido el mal hábito sino también a familiares a quienes se les denomina fumadores pasivos por el simple hecho de inhalar el humo, lo que es perjudicial para el aspecto de la piel y para la salud. 
Rev. SINAPSIS, Vol. 7, № 2, diciembre 2015

Se pudo concluir además, que las arrugas de los fumadores son diferentes de las de los no fumadores son más estrechas, profundas y con contornos bien marcados. Los fumadores de 40 a 49 años tienen una probabilidad de arrugas idéntica a la de los no fumadores de 60 o 70. El consumo de tabaco provoca deshidratación de la piel que aparece áspera, quebradiza e inflexible. A esto se suma la poca oxigenación de las células, que hace que el cutis parezca grisáceo y apagado. Las personas privadas de la libertad no tienen un cuidado oportuno en su piel, como lo haría cualquier persona libre.

De acuerdo a las consideraciones finales del trabajo, se determinó necesaria la elaboración de un material educativo dirigido a las internas de los Centros de Privación de la libertad, por lo cual se socializó con las autoridades para entregar un tríptico con información relevante acerca de los efectos negativos del consumo del tabaco 
Rev. SINAPSIS, Vol. 7, Nº 2, diciembre 2015

\section{Bibliografía}

1. Alvares, FJ. (2013). El tabaquismo cómo problema de salud pública, Promoción y Educación para la Salud. Madrid, capitulo 1 p.32.

2. Andraka, P. (2011). El envejecimiento de la piel. Trampas al cigarro. Estados Unidos de América: Palibrio.

3. Becoña, E. (2010). Nicotina. Dependencia del Tabaco. Madrid: Sociología Española de Psicología Clínica, Legal y Forence.

4. Branco, C. (2010). Envejecimiento de la piel. Envejecimiento de la piel y las mucosas. Buenos Aires: Médica Panamericana S.A

5. Cornejo, J. (2013). Efectos nocivos de los vicios en la piel. [En línea]. Consultado: [20, noviembre, 2013] Disponible en: www.macroestetica.com /artículos/efectosnocivos-de-los-vicios-en-la-piel/

6. Fuente, R. (2012). Componentes del humo del tabaco. El papel determinante del tabaco. Madrid: Díaz de Santos.

7. López, A. (2014). El tabaquismo: consecuencias en dermatología y estética. Recuperado en 23 de marzo de 2015 en http://www.seme.org/area_pro/textos_articulo.php?id=5

8. Lucán, M. (2011). El tabaco. La protección del consumidor frente a los daños. Madrid: Reus s.a

9. Radicales Libres, publicado el 20 de abril de 2013 y recuperado el 20 de noviembre de 2013 mediante http/http://www.ojocientifico.com/4335/que-son-losradicales-libres\#5355

10. Rodrigues, O. (2010). El tabaco terapia sexual. Sao Paulo: Fevereiro.

11. Sauvy, A. (1966) Théorie Générale de la Population, Paris: P. U. F 